\title{
Percepção de extrativistas sobre os buritizais na região dos Lençóis Maranhenses, Brasil
}

\section{Perception of extractivists about the Mauritia flexuosa palm swamp in the Lençóis Maranhenses Region, Brazil}

\author{
Irlaine Rodrigues Vieira ${ }^{1}$ \\ Jefferson Soares de Oliveira ${ }^{2}$ \\ Geisiane Oliveira Silva ${ }^{3}$ \\ Kelly Polyana Pereira dos Santos ${ }^{4}$ \\ Fábio José Vieira ${ }^{5}$ \\ Roseli Farias Melo de Barros ${ }^{6}$
}

\begin{abstract}
Resumo
Pesquisas de percepção ambiental evidenciam o contexto extrativista e auxiliam na elaboração de políticas ambientais. Apesar dos benefícios sociais e econômicos, a intensificação do extrativismo pode promover a insustentabilidade ecológica e alteração da percepção dos valores florestais conservacionistas. Na região dos Lençóis Maranhenses, Nordeste do Brasil, é crescente a venda de produtos extraídos da palmeira Mauritia flexuosa L.f. para abastecer mercado local e nacional. Desta forma, o presente trabalho objetivou avaliar quais valores florestais são percebidos pelos extrativistas em municípios da região dos Lençóis Maranhenses e como o perfil socioeconômico interfere na percepção ambiental dos entrevistados. A pesquisa foi conduzida por meio de formulários semiestruturados aplicados a 271 extrativistas dos município de Barreirinhas, Paulino Neves e Tutóia. Os valores florestais percebidos foram divididos em três categorias: econômica, ecológica e social. Os dados obtidos revelaram que houve uma priorização da categoria econômica, com destaque para o valor florestal fonte de renda. Em Barreirinhas, os valores alimentação e regulação do clima estiveram relacionados a características socioeconômicas tais como gênero e renda mensal proveniente da venda do artesanato. Há o conhecimento sobre os danos ambientais que ocorrem nos buritizais, entretanto não existe uma efetiva participação das comunidades na gestão das Unidades de Conservação ambientais da região.
\end{abstract}

Palavras-chave: Valores florestais. Gestão ambiental. Unidades de Conservação. Renda.

\footnotetext{
1 Universidade Federal do Piauí, Parnaíba, Piauí, Brasil. irlainervieira@gmail.com

${ }^{2}$ Universidade Federal do Piauí, Parnaíba, Piauí, Brasil. jefferson.oliveira@gmail.com

${ }^{3}$ Universidade Federal do Piauí, Parnaíba, Piauí, Brasil. aneoliveirac@hotmail.com

${ }^{4}$ Universidade Estadual do Piauí, Corrente, Piauí, Brasil. kellypolyana@hotmail.com

5 Universidade Estadual do Piauí, Picos, Piauí, Brasil. fabao.bio@hotmail.com

${ }^{6}$ Universidade Federal do Piauí, Teresina, Piauí, Brasil. rbarros.ufpi@gmail.com

Artigo recebido em: 14/06/2017. Aceito para publicação em: 09/07/2019.
} 


\begin{abstract}
Researches of environmental perception evidence the extractive context and help in the elaboration of environmental policies. Despite the social and economic benefits, the intensification of extractivism can promote ecological unsustainability and alteration in the perception of forest conservation values. In the region of Lençóis Maranhenses, Northeast Brazil, the sale of products extracted from Mauritia flexuosa L.f palm to supply the local and national market is increasing. Thus, the present work aimed to evaluate the forest values perceived by extractivists in municipalities of the region of Lençóis Maranhenses and how the socioeconomic profile interfere with the environmental perceptions of the interviewees. The research was conducted through semi-structured forms applied to 271 extractivists from the municipality of Barreirinhas, Paulino Neves and Tutóia. The perceived forest values were divided into three categories: economic, ecological and social. It was contacted that there was a prioritization of the values included in the Economic category, in which the source of income is highlighted in the municipalities that most extract the palms. The data obtained revealed that there was a prioritization of the economic category, with emphasis on the forest value source of income. In Barreirinhas, food and climate regulation values were related to socioeconomic characteristics such as gender and monthly income from the sale of handicrafts. There is knowledge about the environmental damages that occur in the forest, however there is no effective participation of the communities in the management of the Environmental Conservation Units of the region.
\end{abstract}

Keywords: Forest values. Environmental management. Conservation Units. Income.

\title{
Introdução
}

É desafiador conciliar a conservação ambiental ao desenvolvimento econômico diante da crescente demanda por recursos naturais, até mesmo em áreas ambientalmente protegidas (LAURANCE, 2012). Residentes no interior ou proximidades das Unidades de Conservação ambiental, especialmente comunidades menos favorecidas economicamente, dependem fortemente dos recursos florestais para a alimentação e como fonte de renda (VEDELD, 2012; THONDHLANA e MUCHAPONDWA, 2014), o que pode resultar em danos ao meio ambiente (BENNETT e DEARDEN, 2014) ou contribuir com a sua conservação (VIEIRA et al., 2016). As preferências de utilização dos recursos naturais dependem das percepções (ADAMOWICZ et al., 1997), as quais podem ser influenciadas por fatores socioeconômicos 
como idade (YANG et al., 2015); gênero (ALLENDORF e YANG, 2013) e renda (SHARAUNGA et al., 2015).

Estudos de percepção ambiental auxiliam na formulação de uma correta gestão. Estas pesquisas esclarecem a realidade em que ocorre o extrativismo e orienta na elaboração de medidas políticas em áreas ambientalmente protegidas (ALLENDORF e YANG, 2013). Neste contexto, destacamos as áreas de proteção ambiental inseridas na região dos Lençóis Maranhenses. Nestas áreas há a presença de florestas de Mauritia flexuosa L.f. (BRASIL, 2002) que é crescentemente extraída para a extração de fibras destinadas para a confecção de artesanatos que abastecem o mercado turístico local e nacional (IPEA, 2016).

O extrativismo de produtos florestais destinado ao comércio tende a impulsionar o desenvolvimento econômico local (SAYER et al., 2012) e favorece o sustento de comunidades socioeconomicamente vulneráveis que possuem acesso aos recursos florestais (VIRAPONGSE et al., 2014). Apesar do favorecimento social e econômico, a intensificação do extrativismo pode trazer consequências negativas (BELCHER e SCHRECKENBERG, 2007), como conflitos sociais, insustentabilidade ecológica (SCHMINK, 2004) e alteração da percepção dos valores florestais conservacionistas (VIEIRA e LOIOLA, 2014). É neste contexto que este estudo foi realizado, objetivandose investigar a percepção ambiental de comunidades situadas em uma realidade de extração destinada ao comércio e avaliar como o perfil socioeconômico interfere nisto.

\section{Procedimentos e pesquisa}

\section{Área de estudo}

O presente estudo foi realizado nos municípios de Barreirinhas, Paulino Neves e Tutóia, os quais compõem a cadeia produtiva do artesanato na região dos Lençóis Maranhenses, Nordeste do Brasil, Estado do 
Maranhão (IPEA, 2016). Nestes municípios selecionaram-se as comunidades extrativistas que abastecem as associações de artesãos e extrativistas em cada município avaliado: Residencial Brasil, Cebola, Ladeira e Barreiro, no município de Barreirinhas, Água Riquinha, município de Paulino Neves, e Justa no município de Tutóia.

A área de estudo abrange duas Áreas de Proteção Ambiental Estaduais, a Upaon-Açu / Miritiba / Alto Preguiças e a Boca do Rio Preguiças / Pequena Região Lençóis / Lagunar, as quais se situam na região dos Lençóis Maranhenses, Nordeste Brasileiro (ICMBIO, 2008).

A vegetação é caracterizada como restingas, vegetação costeira geralmente associada a manguezais, campos de dunas e florestas de galeria de água doce (ICMBIO, 2008). As florestas de galeria de água doce é habitada pela palmeira Mauritia flexuosa L.f. conhecida popularmente como buriti no Brasil, formando o buritizal (BRASIL, 2002).

O Estado do Maranhão é o maior extrativista de fibras da palmeira buriti no Nordeste brasileiro e na região dos Lençóis Maranhenses a coleta é uma das principais atividades extrativistas, contribuindo significativamente para a renda do local (IPEA, 2016). Os municípios de Barreirinhas (103 toneladas/ano em 2014) e Tutóia (38 toneladas/ano em 2014) se destacam como um dos maiores extrativistas do Brasil (IBGE, 2014). Paulino Neves, apesar de não aparecer nas estatísticas do IBGE, tem o extrativismo vegetal de buriti como fonte de renda familiar (IPEA, 2016).

\section{Coleta dos dados}

Os extrativistas de Barreirinhas, Paulino Neves e Tutóia foram identificados através de amostragem Bola de neve e realizado entrevistas semiestruturadas com todos os indicados nas comunidades (ALBUQUERQUE et al., 2014). 
As entrevistas semiestruturadas foram realizadas ao longo de um ano e quatro meses entre agosto de 2014 e dezembro de 2015. A todos os entrevistados foi solicitado a assinatura no termo de consentimento livre e esclarecido. Este estudo foi aprovado pelo Comitê de Ética em Pesquisa da Universidade Federal do Piauí, com o número de permissão 886.193.

Os formulários foram compostos por três partes. Na primeira foram levantadas as variáveis socioeconômica gênero, idade, escolaridade, renda mensal somente obtida pela comercialização de produtos extraídos e confeccionados com o buriti e renda mensal total, correspondente ao somatório de toda a renda mensal obtida. A segunda parte se baseou na listagem sobre a percepção da importância do buritizal (Valores florestais). A terceira teve por objetivo avaliar o conhecimento sobre a Unidade de Conservação no qual são residentes e as problemáticas ambientais. Para tanto realizou-se quatro questionamentos: $1^{\circ}$ Você conhece alguma Unidade de Conservação ambiental? Caso a resposta fosse positiva, questionava-se quais e porque a área é considerada uma Unidade de Conservação ambiental. $2^{\circ}$ Você participa da gestão da Unidade de Conservação ambiental? $3^{\circ} \mathrm{O}$ que prejudica os buritizais por aqui? $4^{\circ}$ As áreas de buritizais estão sendo reduzidas? Caso afirmativo, questionava-se as causas.

\section{Análise de dados}

Os valores citados pelos informantes foram agrupados em três categorias de acordo com o tipo de valor relatado (Tabela 1), seguindo a metodologia adotada por Yang et al. (2015). 
Tabela 1. Categorização dos valores florestais de acordo com a descrição dos informantes da região dos Lençóis Maranhenses, Maranhão, Brasil.

\begin{tabular}{|c|c|c|}
\hline Categoria & Valor florestal & Descrição \\
\hline \multirow{5}{*}{ Econômica } & Alimentícios & Recursos alimentícios humanos e animais. \\
\hline & $\begin{array}{l}\text { Utensílios } \\
\text { domésticos }\end{array}$ & $\begin{array}{l}\text { Utensílios confeccionados que auxiliam na rotina diária como } \\
\text { cestas, tecelagens, peneiras, artefatos de caça e gaiolas. }\end{array}$ \\
\hline & Madeira & $\begin{array}{c}\text { Madeira para cozinhar e para construção de móveis, portas, } \\
\text { cercas, casas, pontes, barcos. }\end{array}$ \\
\hline & Fonte de Renda & $\begin{array}{c}\text { Extrativismo de recursos florestais destinado exclusivamente } \\
\text { para a venda e obtenção de renda, como a produção dos } \\
\text { artesanatos turísticos. }\end{array}$ \\
\hline & Recursos naturais & $\begin{array}{l}\text { Outras utilidades dos recursos naturais, como a cobertura de } \\
\text { casas e adubo. }\end{array}$ \\
\hline \multirow{7}{*}{ Ecológica } & $\begin{array}{l}\text { Manutenção da } \\
\text { biodiversidade }\end{array}$ & $\begin{array}{c}\text { Manutenção da biodiversidade florestal como abrigo e nidificação } \\
\text { de animais. }\end{array}$ \\
\hline & Fonte de água & $\begin{array}{c}\text { Obtenção de água para irrigação, limpeza em geral, banho, cocção } \\
\text { de alimentos, hidratação humana e animal. }\end{array}$ \\
\hline & Purificação da água & $\begin{array}{c}\text { Purificação da água para o consumo, tornando-a insípida, inodora } \\
\text { e apropriada para a utilização. }\end{array}$ \\
\hline & Purificação do ar & Purificação do ar e redução da poeira, $\mathrm{CO}^{2}$ e poluentes. \\
\hline & Proteção do solo & As raízes evitam a erosão do solo. \\
\hline & $\begin{array}{l}\text { Manutenção das } \\
\text { margens dos rios }\end{array}$ & A floresta previne o deslizamento de terras das margens dos rios. \\
\hline & Regulação do clima & Ajuste no microclima, temperatura mais amenas. \\
\hline \multirow[b]{2}{*}{ Social } & Beleza & Contemplação e valorização da beleza florestal. \\
\hline & Valores culturais & $\begin{array}{c}\text { Uso da floresta para confeccionar roupas de danças típicas, como } \\
\text { a "bumba meu boi" e confecção de esteiras para as gestantes } \\
\text { parir. }\end{array}$ \\
\hline
\end{tabular}

Org.: Adaptado de Yang et al., 2015.

Para verificar se haviam diferenças entre os valores florestais percebidos entre os municípios, foi contado o número de vezes que o valor foi citado pelos informantes. As diferenças no número de valores florestais por informante entre os municípios foram avaliadas utilizando os testes de Kruskal Wallis, com posterior utilização do teste de Student-NewmanKeuls.

Análises de regressão linear binária multivariada (R2) foram utilizadas para analisar se as variáveis independentes gênero, idade, anos de estudo, renda familiar mensal (rendimento de todos os membros da família por mês) e rendimento mensal da comercialização de $M$. flexuosa explicam a percepção ou não do valor florestal. Além disso, a análise de variância ANOVA foi realizada para avaliar a variabilidade da percepção 
entre fatores socioeconômicos que mostraram influência. Os testes estatísticos foram realizados utilizando o software BioEstat 5.0 (AYRES et al., 2007). Valores F de significância e p (p-valor) menores que 0,05 foram considerados significativos para análise.

\section{Resultados e discussão}

\section{Valores florestais percebidos}

As entrevistas foram realizadas com 271 informantes (202 mulheres e 69 homens) cuja idade variou entre 18 a 89 anos. Foram registrados a percepção de 14 valores florestais, cinco categorizados como valores econômicos, sete valores ecológicos e dois sociais (Tabela 2). Destacou-se a categoria econômica, na qual o valor florestal fonte de renda foi o mais percebido nos três municípios avaliados, não havendo diferenças estatísticas significativas entre Tutóia e Barreirinhas $(p<0.05)$ quanto a frequência de citação (Tabela 2). Dentre os valores da categoria ecológica, a manutenção da biodiversidade foi percebida somente em Barreirinha, enquanto a purificação do ar e da água foram descritos somente em Tutóia (Tabela 2). Não foram observadas diferenças significativas para os demais valores florestais citados $(\mathrm{p}>0,05)$. 
Tabela 2. Média da frequência dos valores florestais percebidos sobre buritizais nos municípios de Barreirinhas, Paulino Neves e Tutóia, Maranhão, Brasil.

\begin{tabular}{|c|c|c|c|c|c|}
\hline \multicolumn{2}{|c|}{ Valores florestais } & \multicolumn{4}{|c|}{ Média de citação dos valores florestais percebidos } \\
\hline Valo & $s$ florestais & Barreirinhas & Paulino Neves & Tutóia & $\begin{array}{c}\text { p- } \\
\text { valor }\end{array}$ \\
\hline \multirow{5}{*}{ Econômicos } & Alimentação & $0,344 \pm 0,647^{\mathrm{a}}$ & $0,649 \pm 0,482 \mathrm{~b}$ & $0,533 \pm 0,507 \mathrm{~b}$ & 0,0001 \\
\hline & $\begin{array}{l}\text { Utensílios } \\
\text { domésticos }\end{array}$ & $0,016 \pm 0,126^{\mathrm{a}}$ & $0,167 \pm 0,377 \mathrm{a}$ & $0,461 \pm 0,508 b$ & 0,0007 \\
\hline & $\begin{array}{l}\text { Madeira para } \\
\text { construção }\end{array}$ & $0,042 \pm 0,202^{\mathrm{a}}$ & $0,125 \pm 0,334 \mathrm{a}$ & $0,500 \pm 0,511 b$ & 0,0007 \\
\hline & Recursos naturais & $0,069 \pm 0,254$ & $0,227 \pm 0,422$ & $0,000 \pm 0,000 *$ & 0,1740 \\
\hline & Fonte de renda & $0,862 \pm 0,375^{\mathrm{a}}$ & $0,660 \pm 0,479 \mathrm{~b}$ & $0,813 \pm 0,397 \mathrm{a}$ & 0,0070 \\
\hline \multirow{7}{*}{ Ecológicos } & $\begin{array}{l}\text { Manutenção da } \\
\text { biodiversidade }\end{array}$ & $0,021 \pm 0,144$ & $0,000 \pm 0,000 *$ & $0,000 \pm 0,000 *$ & 0,9620 \\
\hline & Fonte de água & $0,005 \pm 0,073$ & $0,098 \pm 0,300$ & $0,188 \pm 0,397$ & 0,1960 \\
\hline & Purificação da água & $0,000 \pm 0,000^{*}$ & $0,000 \pm 0,000 *$ & $0,125 \pm 0,336$ & 0,5170 \\
\hline & Purificação do ar & $0,000 \pm 0,000^{*}$ & $0,000 \pm 0,000 *$ & $0,031 \pm 0,177$ & 0,9610 \\
\hline & Proteção do solo & $0,010 \pm 0,103$ & $0,020 \pm 0,141$ & $0,074 \pm 0,267$ & 0,8670 \\
\hline & $\begin{array}{l}\text { Manutenção das } \\
\text { margens de rios }\end{array}$ & $0,016 \pm 0,176$ & $0,039 \pm 0,196$ & $0,063 \pm 0,246$ & 0,9110 \\
\hline & Regulação do clima & $0,005 \pm 0,073$ & $0,039 \pm 0,196$ & $0,156 \pm 0,369$ & 0,3900 \\
\hline \multirow{2}{*}{ Sociais } & Beleza & $0,016 \pm 0,125$ & $0,04 \pm 0,198$ & $0,000 \pm 0,000 *$ & 0,9490 \\
\hline & Valores culturais & $0,032 \pm 0,176$ & $0,000 \pm 0,000^{*}$ & $0,000 \pm 0,000^{*}$ & 0,9180 \\
\hline
\end{tabular}

Valores são expressos em média de frequência de resposta \pm DP, analisado pelo teste Kruskal Wallis e seguido por Student Newman-Keuls. Letras diferentes indicam presença de diferença estatística $(<$ 0,05). *Valor florestal não citado.

Org.: do Autor, 2017.

Observamos que as diferentes categorias de valores percebidos (econômica, ecológica e social) foram semelhantes àquelas registradas em outras Unidades de Conservação como nos estudos desenvolvidos por Sodhi et al. (2009) na indonésia, Myanmar, Filipinas e Tailândia e por Yang et al. (2015) na China. Diferentemente do nosso trabalho, estes autores não verificaram a priorização do valor florestal fonte de renda.

De acordo com Steel et al. (1994) e Sharaunga et al. (2015), o ambiente de trabalho e a dependência financeira extrativista podem orientar para uma visão mais antropocêntrica dos valores florestais percebidos. Assim, para os municípios avaliados, o contexto extrativista nos 
quais os informantes se encontram inseridos pode determinar uma percepção da categoria econômica e a priorização do valor fonte de renda entre os entrevistados.

\section{A influência das variáveis socioeconômicas na percepção dos valores florestais}

As variáveis socioeconômicas não estiveram relacionadas a percepção dos valores florestais em Tutóia e em Paulino Neves (F de significação < 0,05) enquanto que em Barreirinhas o gênero esteve relacionado a percepção do valor florestal alimentício $\left(F=0,013 ; R^{2}: 0,075 ; p=0,022\right)$ e a renda proveniente da venda do artesanato à percepção da regulação do clima $(\mathrm{F}<$ 0,$\left.01 ; R^{2}=0,148 ; p=0,001\right)$.

Em Barreirinhas, as mulheres mencionaram mais vezes o valor florestal alimentício que os homens $(p<0,05)$ (Figura 1A). De modo diferente, outros trabalhos, como os estudos realizados por Xu et al. (2006) na China e por Allendorf e Yang (2013) no mesmo país, descreveram que os homens tendem a conhecer mais sobre os valores florestais. Além disso, estes valores são mais antropocêntricos em comparação com o gênero feminino (MCFARLANE e BOXALL, 2000; SHARAUNGA et al., 2015). No presente trabalho, a maior percepção do valor florestal alimentício entre as mulheres pode estar associado ao fato delas serem responsáveis por preparar o alimento para a família.

Verificamos também que a renda mensal proveniente do artesanato teve correlação com o valor regulação do clima em Barreirinhas $(p=0,001)$ e foi maior entre os que recebem entre $R \$ 101,00$ e $R \$ 200,00$ reais por mês com a venda de artesanato quando comparado aos que recebem entre $R \$$ 1,00 e $\mathrm{R} \$ 100,00$ reais por mês (Figura $1 \mathrm{~B}$ ). 
Figura 1- Avaliação da influência dos fatores socioeconômicos na percepção dos valores florestais no município de Barreirinhas, Maranhão, Brasil. A: gênero (M: Masculino e F: Feminino). B: Renda mensal do artesanato. * e linhas indicam diferença significativa entre os grupos. Os dados foram avaliados por análise de variância binária utilizando o teste de ANOVA $(p<0.05)$.
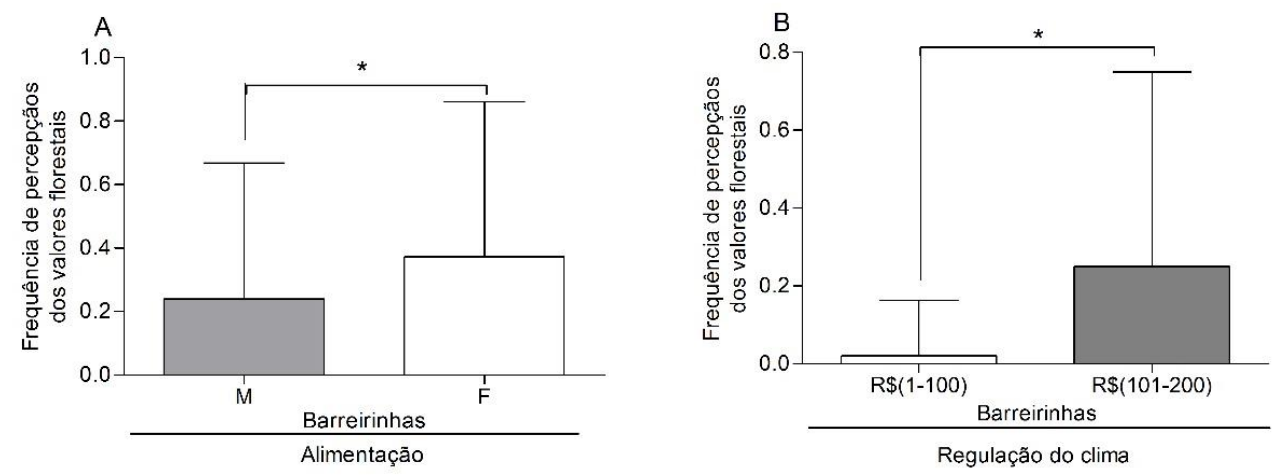

Fonte: Org. dos autores

Os estudos desenvolvidos por Sodhi et al. (2009) e Sharaunga et al. (2015) comprovam a relação entre a renda proveniente do derivado florestal e a percepção sobre o meio ambiente. Segundo Sharaunga et al. (2015) os benefícios financeiros obtidos de derivados florestais tendem a orientar a percepção, principalmente, para os valores da categoria econômica, enquanto que pessoas que obtêm rendas maiores de fontes não florestais detêm orientações menos antropocêntricas, por depender menos da floresta. Estes resultados foram diferentes dos observados para a região dos Lençóis Maranhenses. Não houve correlação entre a renda proveniente do artesanato e a percepção de valores da categoria econômica, mas sim para a categoria ecológica.

Verificamos que em Barreirinhas, a renda obtida pela venda de produtos extraídos do buriti influenciou na percepção do valor regulação do clima. Foi relatado, entre os entrevistados que mais vendem, a necessidade de adentrar frequentemente os buritizais para extrair recursos para a obtenção de renda. Nestas áreas sentem que o clima é mais ameno que em 
áreas urbanizadas, o que pode proporcionar a percepção deste valor entre os informantes com maior obtenção de renda derivada do extrativismo.

\section{Compreensão sobre as Unidades de Conservação (UC)}

Os informantes dos três municípios foram questionados se os buritizais são Unidades de Conservação (UCs). No município de Barreirinhas, 43,92\% (83) dos informantes não as compreendem como uma UC, 25,93\% (49) não souberam responder e 30,16\% (57) afirmaram que sim. Entre os informantes que afirmaram saber que os buritizais são Unidade de Conservação ambiental, 17 não souberam explicar o porquê de ser uma UC, e 29 acreditam que é devido à presença de órgãos fiscalizadores. Os demais atribuíram à presença do rio (8) e ao buritizal (3).

No município de Paulino Neves, 76\% (38) acreditam que os buritizais não são uma UC; $22 \%$ (11) não souberam responder e $2 \%$ (1) afirmaram que sim, mas não soube explicar o porquê.

Já em Tutóia, 15,63\% (5) afirmam que não são uma Unidade de Conservação ambiental, 71,88\%, (23) não souberam responder e 12,50 \% (4) relataram que sim. Dentre os que afirmaram saber que o buritizal é uma UC, um informante não soube explicar o porquê de ser uma UC, um associou à presença de órgãos ambientais do governo fiscalizadores e dois associaram a proteção do rio. Nenhum dos entrevistados nos três municípios afirmou participar da gestão.

É comum que pessoas residentes em Unidades de Conservação saibam sobre a sua existência (BENNETT e DEARDEN, 2014; YANG et al., 2015). Entretanto, nossos resultados demonstraram que a maioria dos informantes desconhece que residem em uma e os que estão cientes não sabem descrever porque ela é considerada assim. Esta informação é 
semelhante às registradas por Xu et al. (2006) e Dias et al. (2007) quanto a deficiência na compreensão dos residentes nestas áreas. Xu et al. (2006) e Vieira e Loiola (2014) associam esta deficiência a escassa comunicação entre os habitantes e os órgãos gestores, o que limita o conhecimento da população e consequentemente a participação na gestão da Unidade de Conservação no qual estão inseridos.

Quando questionados o que prejudica os buritizais, $15 \%$ (28) dos entrevistados em Barreirinhas relataram a extração de madeira; 71\% (136) a extração de folhas imaturas da palmeira buriti para a confecção de artesanatos destinados à venda para os turistas, 8,46\% (16) a devastação pela especulação imobiliária promovida pela rede hoteleira, 2,66\% (5) as queimadas, $1,08 \%$ (2) a estiagem e 1,8\% (2) a ação de pássaros (urubus). Em Paulino Neves, 38\% (19) dos entrevistados atribuíram à queima e devastação (coivara), 22\% (11) ao intenso extrativismo de folhas imaturas dos buritis, 20\% (10) a causas naturais como escassez hídrica, raios, envelhecimento da planta; 14\% (7) não souberam responder, 6\% (3) ao desmatamento para a agricultura. Em Tutóia, 40,64\% (13) atribuem à prática de coivara, 28,13\% (9) à extração de folhas imaturas do buriti, $28,13 \%$ (9) a causas naturais como herbivoria, escassez hídrica, envelhecimento da planta e 3,1\% (1) à pecuária.

Quando questionados se as áreas de buritizais estão sendo reduzidas 89,43\% (169) dos informantes de Barreirinhas afirmam que está havendo uma redução principalmente pela morte dos buritis que são intensamente explorados, 8,99\% (17) responderam não e 1,58\% (3) não souberam responder. Alguns informaram que há a necessidade de se deslocar para o município de Paulino Neves para obter folhas imaturas de buriti. De modo semelhante, em Paulino Neves 50\% (25) dos informantes afirmaram que está havendo a redução de seus buritizais principalmente ocasionada pela morte da palmeira promovida por extrativistas que veem de Barreirinhas. Os demais informantes, afirmaram que há uma redução dos buritizais 
devido à estiagem que ocorre no local (12\% - 6) e 38\% (19) relataram que não está acontecendo uma redução das áreas de buritizais. Este dado demonstra que o manejo tradicional de extrativismo voltado para o comércio, não é ambientalmente viável em Barreirinhas e em Paulino Neves.

Em Tutóia, os entrevistados não verificaram uma redução nas áreas de buritizais. Em um estudo desenvolvido por Vieira et al. (2016), foi constatada a crença na punição por elementos místicos aos que danificam o meio ambiente, o que contribui para a conservação dos buritizais neste município.

\section{Considerações Finais}

O ambiente de trabalho e a dependência financeira extrativista contribuem para uma visão antropocêntrica dos valores florestais com a priorização da categoria econômica e destaque para o valor florestal fonte de renda. Apesar disso, uma maior renda obtida por meio da venda dos recursos extraídos não é um fator determinante para a percepção da categoria econômica, no qual pessoas que obtêm maiores rendas de fontes florestais apresentaram priorização de percepções ecológicas. A influência do perfil sócio econômico na percepção é particular de cada grupo, mesmo estando inseridos no mesmo contexto extrativista. A compreensão dos moradores da unidade de conservação ambiental sobre a existência e o porquê dessas áreas serem consideradas assim é deficiente. Isto contribui para que práticas extrativistas nessas áreas possam ser ambientalmente inviáveis.

\section{Agradecimentos}


Os autores são gratos às comunidades Cebola, Ladeira, Residencial Brasil, Barreiro, Água Riquinha e Justa; às presidentes das Associações de Artesãos e Extrativistas de Tutóia e Paulino Neves e a da Cooperativa de Artesão e Extrativistas de Barreirinhas; à todos os entrevistados e suas famílias pela receptividade e à Capes (Coordenação de Aperfeiçoamento de Pessoal de Nível Superior) pela concessão de bolsa de estudo a primeira autora.

\section{Referências}

ALBUQUERQUE, U. P.; RAMOS, M. A.; LUCENA, R. F. P.; ALENCAR, N. L. Methods and techniques used to collect ethnobiological data. In: ALBUQUERQUE, U. P.; CUNHA, L. V. F. C.; LUCENA, R. F. P.; ALVES, R. R. N. (Eds.). Methods and techniques in ethnobiology and ethnoecology. New York: Springer, 2014. p. 15-37. https://doi.org/10.1007/978-1-4614-8636-7_2

ADAMOWICZ, W.; SWAIT, J.; BOXALL P.; LOIVIEREE J. WILLIAMS M. Perceptions versus objective Meassures of environmental quality in combined revealed and stated preference models af environmental valuation. Journal of environmental economics and management, v.32, n. 1, p. 65-84, 1997. https://doi.org/10.1006/jeem.1996.0957

ALLENDORF, T.; YANG, J. The role of ecosystem services in park-people relationships: the case of Gaoligongshan Nature Reserve in Southwest China. Biological Conservation, v. 167 , n. 1, p. 187-193, 2013. https://doi.org/10.1016/j.biocon.2013.08.013

AYRES, M.; AYRES, J. R. M.; AYRES D. L.; SANTOS A. S. Bioestat 5.0: aplicações estatísticas nas áreas das ciências biológicas e médicas. Belém: Sociedade Civil Mamirauá, 2007.

BELCHER B.; SCHRECKENBERG, K. Commercialisation of non-timber forest products: a reality check. Development Policy Review, v. 25, n.3, p.355-377, 2007. https://doi.org/10.1111/j.1467-7679.2007.00374.x

BENNETT, N.J.; DEARDEN, P. Why local people do not support conservation: Community perceptions of marine protected area livelihood impacts, governance and management in Thailand. Marine Policy, v.44, n. 1, p. 107-1016, 2014. https://doi.org/10.1016/j.marpol.2013.08.017

BRASIL. Resolução Conama $n^{\circ} 303$ de 20 de março de 2002. Disponível em: < http://www.mma.gov.br/port/conama/res/res02/res30302.html>. Acesso em: 22 de abril de 2016.

DIAS, T.L.P.; ROSA, R.S.; DAMASCENO, L.C.P. Aspectos socioeconômicos, percepção ambiental e perspectivas das mulheres marisqueiras da Reserva de Desenvolvimento Sustentável Ponta do Tubarão (Rio Grande do Norte, Brasil). Gaia Scientia, v.1, n.1, p. 2535, 2007.

IBGE - Instituto Brasileiro de geografia e estatística. Produção da Extração Vegetal e da Silvicultura. Quantidade produzida na extração vegetal, por tipo de produto extrativo. Municípios-UF. 2014. Disponível em: $<$ http://www.sidra.ibge.gov.br/bda/tabela/protabl.asp?c=289\&i=P\&nome=on\&qtu $8=136 \&$ not arodape $=0$ on $\&$ tab $=289 \&$ opn $8=0 \&$ unit $=0 \&$ pov $=1 \& o p n 1=2 \& O p c$ TipoNivt $=2 \& n i v t=0 \& \sec 193=$ 3424\&orp $=4 \& q$ tu $3=26 \&$ orv $=2 \&$ opc $193=1 \& q$ tu $2=5 \&$ opv $=1 \&$ pop $=1 \&$ opn $2=0 \&$ sev $=144 \&$ opp $=$ f $1 \&$ opn $3=0 \& q$ tu $6=5204 \&$ poc $193=1 \& q t u 1=1 \&$ opn $9=0 \&$ cabec $=$ on $\&$ orc $193=3 \&$ ascendente $=$ on \&sep $=56308 \&$ orn $=1 \&$ pon $=2 \& q t u 9=548 \&$ opn $6=0 \&$ digt $6=\& 0$ pcCara $=87 \&$ proc $=1>. \quad$ Acesso em: 01 october. de 2016. 
ICMBIO - Instituto Chico Mendes de Conservação da Biodiversidade. Plano de Manejo do Parque Nacional dos Lençóis Maranhenses. Encarte 4 - Contexto regional. 2008. Disponível em: $<$ http://www.icmbio.gov.br/portal/images/stories/imgsunidadescoservacao/06ENCARTE4.pd f $>$. Acesso em: 28 Junho de 2016.

IPEA- Instituto de Pesquisa Econômica Aplicada. Economias baseadas em biomas: estudo das cadeias de comercialização de produtos florestais não madeireiros na região de planejamento dos lençóis maranhenses. Rio de Janeiro: IPEA, 2016.

LAURANCE, W.F.; USECHE, D.C.; RENDEIRO J. et al . Averting biodiversity collapse in tropical forest protected areas. Nature, v.489, n. 1, p. 290-294, 2012. https://doi.org/10.1038/nature11318

MCFARLANE, B. L.; BOXALL, P. C. Factors influencing forest values and attitudes of two stakeholder groups: the case of the Foothills Model Forest, Alberta, Canada. Society \&
Natural
Resources, v. 13,
n.
7
p.649-661,
2000.

https://doi.org/10.1080/08941920050121927

SAYER, J.A.; ENDAMANA, D.; RUIZ, P.M.; BOEDHIHARTONO, A.K.; NZOOH, Z.; EYEBE, A.; AWONO, A.; USONGO, L. Global financial crisis impacts forest conservation in Cameroon. International Forestry Review, v.14, n.1, p.90-98, 2012. https://doi.org/10.1505/146554812799973172

SCHMINK, M. Communities, forests, markets, and conservation. In: ZARIN, D.J.; ALAVALAPATI, J.R.R.; PUTZ F.J.; SCHMINK,M. (Eds.). Working forests in the tropics: conservation through sustainable management? New York: Columbia University Press, 2004. p. 119-129. https://doi.org/10.7312/zari12906-009

SHARAUNGA, S.; MUDHARA, M.; WALE, E. Z. Factors influencing forest value orientations among rural households in KwaZulu-Natal, South Africa. Agroforestry Systems, v. 89, n. 6, p. 943-962, 2015. https://doi.org/10.1007/s10457-015-9827-5

SODHI, N. S.; LEE, T. M.; SEKERCIOGLU, C. H.; WEBB, E. L.; PRAWIRADILAGA, D. M.; LOHMAN, D. J.; PIERCE, N. E.; DIESMOS, A. C.; RAO, M.; EHRLICH, P. R. Local people value environmental services provided by forested parks. Biodiversity \& Conservation, v. 19, n. 4, p.1-14, 2009. https://doi.org/10.1007/s10531-009-9745-9

STEEL, B.S.; LIST, P.; SHINDLER, B. Conflicting values about federal forests: A comparison of national and Oregon publics. Society and Natural Resources, v.7, n.2, p. 137-153, 1994. https://doi.org/10.1080/08941929409380852

THONDHLANA, G.; MUCHAPONDWA, E. Dependence on environmental resources and implications for household welfare: Evidence from the Kalahari drylands, South Africa.

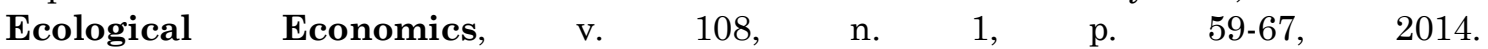
https://doi.org/10.1016/j.ecolecon.2014.10.003

VEDELD, P.; JUMANE, A.; WAPALILA, G.; SONGORWA, A. Protected areas, poverty and conflicts A livelihood case study of Mikumi National Park, Tanzania. Forest Policy and Economics, v.21, n.1, p. 20-31, 2012. https://doi.org/10.1016/j.forpol.2012.01.008

VIEIRA, I.R.; LOIOLA M.I.B. Percepção ambiental das artesãs que usam as folhas de carnaúba (Copernicia prunifera H.E.Moore, Arecaceae) na Área de Proteção Ambiental Delta do Parnaíba, Piauí, Brasil. Sociedade. \& Natureza, v.26, n.1, p. 63-76, 2014. https://doi.org/10.1590/1982-451320140105

VIEIRA, I.R.; OLIVEIRA, J. S. O.; SANTOS, K.P.P.; VIEIRA, F.J.; BARROS, R.F.M. Cosmovisión y etnoconservación en morichales (buritizales), estado de Maranhão, Brasil. Espacios, v. 37, n. 24, p.1-5, 2016.

VIRAPONGSE, A.; SCHMINK, M.; LARKIN, S. Value chain dynamics of an emerging palm fiber handicraft market in Maranhão, Brazil. Forests, Trees and Livelihoods, v. 23, n. 1, p. 1-184, 2014. https://doi.org/10.1080/14728028.2013.868707

XU, J.; CHEN, L.; LU, Y.; FU, B. Local people's perceptions as decision support for protected area management in Wolong Biosphere Reserve, China. Journal of $\begin{array}{lllllll}\text { Environmental Management, } & \text { v. } 78, \quad \text { n. } 4, & \text { p. } 362-372, & 2006 .\end{array}$ https://doi.org/10.1016/j.jenvman.2005.05.003 